\title{
OBSERVATIONS REGARDING THE CONSTANCY OF MUTANTS AND QUESTIONS REGARDING THE ORIGIN OF DISEASE RESISTANCE IN PLANTS ${ }^{1}$
}

\section{PROFESSOR HENRY L. BOLLEY}

\section{Nortif Dakota Agricultural Coliege}

Iт is not my purpose to develop a controversy as to theories, but, in pointing out some features of my studies upon disease resistance, it seems necessary to raise some question concerning the rapid development of the mutation theory which I believe to be worthy of close thought before we accept this theory as replacing, in entirety, the doctrine of evolution as formulated by Darwin.

The DeVriesian school has pointed out one way in which plants and animals originate new individuals with characteristics apparently new. The Mendelian formulas, especially as in late years developed, illustrate clearly how apparently new characteristics may appear to be caused to arise. This would seem to be a fair statement of all that has actually been accomplished.

I assent that most of the conceptions arising from the investigations of Mendel and DeVries are probably correct and, after considering expositions depending upon the experiments of many workers and having experimented sufficiently to understand the meaning of "mutants," "unit characters" and "elementary species," I recognize that these works added much light upon how evolution in plant life takes place, and that henceforth the conception of unit characters must largely form the theoretical working basis for practical breeding work. Yet I feel sure that Darwin's conception was sufficiently broad to embody both features as minor parts of the great concept of organic development. To accept De-

'Read before the American Breeders' Association, Washington, D. C., January 29, 1908. 
Vries' doctrine of mutation as a substitution for the Darwinian conception is, I believe, to accept a part for the whole and to place before the farmer and stockman a doctrine which, if generally accepted as a substitute for the broad conception of Darwin, can not but be narrowing and injurious.

DeVriesian and Mendelian phraseology, in daily use. may be to blame, but in reading many of the late expositions one is led to question whether the doctrine of constancy of elementary species or constancy of unit characters can be accepted by biologists and breeders with any less damage to after progress than that which followed the once complacent acceptance of the Linnæan dogma of the constancy of species.

DeVries, of course, argues for the acceptance of the validity of evolution by mutations, and while one may readily concur that such mutations occur, one who works largely with cereal crops and has always recognized that the individual is the proper starting point for selection work, is apt to be astonished in reading his new work on "Plant Breeding," and falls to questioning whether after all, a mutation may not be merely a "fluctuating variation," big enough and stable enough to be recognized. Is it possible that the nature of these changes is different in kind or only in quantity and range of duration? Does accident play so large a part in plant development and plant breeding as indicated by most DeVriesian writers? One need not object because of the wonderful things said of elementary species, for good Darwinians have always believed in the existence of strains and subspecies which would admit of the name; nor should we expect any less of DeVries than that he should use the arguments related in "Nilson's Discovery" and those from such other experiments and ex. positions as would, when handled in certain lights, tend to show evolution by mutation. Yet, one who recognizes all of the possible merits that accrue from the conception of hypothetical unit characters and of the existence 
of plant strains which admit of the designation "Elementary Species' may be pardoned if he is unable to accept another dogma of constancy, and is unable to subscribe to many DeVriesian conclusions regarding the comparative merits of mutation as opposed to adaptation and natural survival. Most of us, I believe, can only look upon a mutation as one of the types of variation through which the survival process brings about evolution. I believe that we still have to look for the underlying causes. An ardent Darwinian can well agree to the statement: "Species are derived from other species by means of sudden small changes which, in some instances, may scarcely be perceptible to the inexperienced eye, ${ }^{\prime}{ }_{2}$ but may find points of doubt and refuse to follow to the limit when reading a number of statements found in the same work, for example, "From their first appearance they are uniform and constant.", ${ }_{3}$ This statement refers to species. Again, "The conception of mutations agrees with the old view of the constancy of species. This theory assumes that a species has its birth, its lifetime, and its death, even as an individual, and that throughout its life it remains one and the same.", "Each single type (be it species or subspecies or variety) is thus wholly constant from its first appearance and until the time it disappears either after or without the production of daughter species." 5 This last sentence is certainly clear to the effect that a species is never changed, at least so as to affect its after progeny. It is equivalent to saying that all individuals after formation in the seed remain exactly like the parents and, taken with the other statements which insist that plants mutate, means that they remain constant until they change, which is an absurdity in argument. With this, we must assume that a man can recognize ultimate unit characters and can recognize just how many characters it takes to make a species. When

${ }^{2}$ DeVries. Plant Breeding. Page 9.

${ }^{3}$ Ibid., page 9 .

${ }^{4}$ Ibid., page 8.

s Ibid., page 9. 
we can do this, there will not be any use for the Breeders' Association. " . . Mutations occur constantly, without preparation and without intermediates." ${ }_{6}$ I can not think of a mutation or change in species occurring without preparation and without intermediates. It is equivalent to saying without cause. Later, one reads, "We may confidently assume that each single mutation affects only a single unit." "r I would certainly agree with this statement if I could conclude from the author's other statements that he had in mind that a plant might be made up of an inconceivable number of unit characters, the ultimate nature of which may, after all, be only matters of force. "In other words, the principle of adaptation, as one of the main parts of the theory of evolution, should be separated from the study of the geographical distribution. . . . 8 As to this assertion, I agree that plants migrate and select in the same sense that men migrate and select, but I can not agree that a species never adapts itself in such form as to transmit the results of adaptability.

"Environment has only selected the suitable forms from among the throng and has no relation whatever to their origin." "Natural selection . . . causes survival of the fittest; but it is not the survival of the fittest of individuals, but that of the fittest species, by which it guides the development of the animal and vegetable kingdoms." "10

These last two quotations contain sweeping assumptions and all of them taken together must be interpreted as saying that evolution in plant and animal life arises wholly from accidental changes, that is, from mutations which are matters of accident, having no preceding cause, therefore beyond us as breeders to investigate or change. This would naturally deprive agriculturalists

${ }^{6}$ DeVries. Plant Breeding. Page 24.

${ }^{7}$ Ibid., page 322 .

${ }^{8}$ Ibid., page 345 .

${ }^{9} \mathrm{Ibid}$., page 352 .

${ }^{10} \mathrm{Ibid}$., page 9 . 
and stockmen of the right to hope for the adjustment of a species to new surroundings in which at first it does not fit.

It is hard to comprehend a philosophy which recognizes the old conception of species yet breaks it into countless "elementary species," and at the same time claims absolute constancy for the characters of the elementary forms. If mutations, which all agree may occur, do not come from irritations of environment, internal or external, from what cause may they arise? Must we say that each plant with an observable unit character is a species and, with Linnæus, that it always was? My observations do not allow of this thought; neither have I ever seen an accident in nature. The only observed accidents of nature, when known, have always been found to have direct natural causes. I could not work with faith upon plant breeding if I could convince myself that any plant was ever the result of an accident.

The fact that plants mutate and that new types arise in regular mathematical relations due to cross breeding, and that selections from individuals give the correct methods of breeding, seems evident, but I have seen nothing to convince me of absolute constancy of either individual or species. Mutation seems to be just a good name for a grade of natural changes and no more. We have still to look for the causative feature in the environment behind the mutation or change of character; and, if we wish to improve upon agricultural species or varieties or individual strains, we must select from among the occurring changes, whether we call them mutants, elementary species or unit characters. The demonstrations of mutations and the new knowledge that arises from a better understanding of the laws governing union of and correlation of characters must eventually greatly facilitate the studies looking toward an understanding of direct special causes for the changes which occur in the evolution of a type. DeVries' original experiments were of immense value, but I believe his philosophy of constancy to be 
exceedingly bad. There seems to be no observation or experiments which can be interpreted as substantiating' that phase of his writing's. While DeVries' examples all point to the minutest types of variation and change, his philosophy of constancy is directly opposed to this thought and would dash all of the hopes of the average man doing any work looking toward the improvement of strains, or types. Yet, previous to the last few years, since college men have been given sufficient funds for experiment, it was the practical farmer and stockman who had done the work in improvement of agricultural sorts and races in stock, and most of the improvements have been made from pedigreed strains of rather pure type whether we speak of vegetables, fruits, cereals or cattle. I know this statement will meet with objections, but I believe it will be found to stand upon good bases when we remember the work of our horticulturists and best breeders of animals. For example, Wellman, Haynes and Houston used plant gardens for the production of individuals from individual mother plants in wheat before De Vries, Nilson or Hayes worked. I can not convince myself, after studying the results and observing the work of Mr. Haynes in later years, that he or the other two men got results only from their first selections. Each of these men have time over and again told me that they selected only the best strains from the progeny of the best individual and that each year their crop had improved in the direction along which they worked and that it maintained itself reasonably well in the field. This, of course, DeVries would answer was only the result of "fluctuating variations." My studies do not convince me that he is correct. Undoubtedly, there are countless variations which may occur in the field crop which it is folly to assume may be detected and classified as permanent or fluctuating. Certain it is, that Darwin argued for the great stability of certain inbred stocks and that forces of heredity are stronger than those of variation. It is equally true that the longest pursued 
experiments of American agriculture show that pure strains or varieties of close-bred stocks, especially those of cereals, are sufficiently stable to be held to form under intelligent culture, but none of us, as yet, can say further than this. . If we maintain and can agree that a mutation or change has a natural cause and that no plants are stable, constant in character, it is nevertheless not necessary to be assumed that the burden of proof rests entirely upon Darwinians.

What evidence may be placed over against the theory of constant elementary species? Well, as an hypothesis, when carried to the limit, an elementary species becomes a unit character and when unit characters are well known they will as in the case of the units of matter, perhaps become so intelligible as to be recognized as the least conceivable element of natural force which makes for union and which in case of the organic units may make for a heritable change. This, the writer believes, is the ultimate result to which most of DeVries' facts and ob. servations point, and my own observations, together with those of others, as I undestand them, teach that whenever physical and chemical conditions are changed in the least, within or without the plant, some variation will occur in plant substance, and that this may result in a change in the progeny which may be fixed or modified sufficiently to serve agricultural purposes, if the conditions which originate it may be ascertained and reasonably maintained. It is but reasonable that some changes should approach greater stability than others.

The practical breeders of America, years before the work at Amsterdam and Svalof commenced, developed hundreds of varieties; and though I have worked with many varieties and strains of wheat, flax and potatoes and have found them all reasonably stable if given generally stable environment, I have never yet been sure that I have seen a stable plant or stable strain or variety. Some change is continually taking place and any change, I think, may be fixed just in proportion as we know the conditions which originate it. 
This brings me to the statement of a principle of agricultural cropping which, though generally recognized by breeders for years, yet needs to be much emphasized by those who would improve a character of a plant or a plant strain, or maintain a standard of a general crop; namely, the condition under which a character originated or is being originated must be maintained or approached. It is all the more important to hold this feature of cropping well in mind, now that the conception of unit characters and of mutations and of new methods of experimental breeding has proved to be of such fertile aid in the production of new types. Much of the value of agricultural, commercial cropping rests upon the permanence and general use of a few well-maintained or established varieties as against the miscellaneous use of many varieties; thus, for instance, a district or country gains its reputation for a particular crop not by the use of many varieties, as, for example, of wheat, or oranges, but by the use of a few which by careful work are held to their characteristic qualities. It is quite possible to swamp agriculture by the creation of too many forms. Stable industries are built up about reasonably stable crops.

After a new type or a new character in a type has been obtained, new conditions, if they include the essentials of the old conditions, may readily bring about the addition of new features whether we have in mind calling these new features fluctuating or elementary. If the originating conditions are lost or are not maintained, the type should degenerate or retrograde, lose character, and I am quite convinced that it always does. Herein lies the working basis for every-day cropping and breeding. This line of argument is equivalent to saying that socalled fluctuating variations may be maintained and built upon as foundations for important hereditary changes, whether, in writing, we term the resultants mutants, sports or simply elementary units or species. It is equivalent to arguing that the principle of adaptation may 
be active in nature even though mutations are observed. Or, perhaps plainer, it is equivalent to saying that mutations themselves may be nothing more or less than adaptations, adaptive changes. I think breeders who ignore this thought in their work will eventually find themselves without a basis for well-founded experiments.

I have been brought to these conclusions chiefly through careful observations upon my own selection and breeding plots, upon which for a number of years, I have attempted to hasten the survival of the fittest, or of the unlike, through the development of or heightening of the action of plant disease for the purpose of eliminating the weak or unfit. ${ }^{11}$ The method of field work consists essentially in constant culture or cropping to the crop under consideration and in promoting, in every way possible, the development and action of the disease under consideration; and at the same time using all available methods of breeding and selection under these heightened conditions of disease. The method has given marked results when applied to wheat vs. wheat rust; wheat $v s$. wheat smut; flax $v s$. flax rusts; flax $v s$. flax wilt; and potatoes $v s$. scab and blight.

My observations along these lines have been such that I have no fear but that the future will find me right in the assertion (1) that mutants may be so insignificant and numerous as to be unrecognizable and thus fall directly into the class called by DeVries, "fluctuating variations"; or (2) that they may be induced in a mixture of a great number of varieties of a species at one and the same time because of the same environmental causes; or (3) that, in some cases, "fluctuating variations" are of such nature and worth as to allow results to be obtained

11 The full details of the methods and details of the results can not be furnished in the limits of this paper. The method of work has been outlined in the annual reports of the North Dakota Agricultural Experiment Station and in an address delivered before the meeting of farmers and stockmen at St. Louis, October, 1903, and further reported upon in rol. I, page 131 of the report of this association, and also in the Proceedings of the Lansing Meeting of the Society for Promotion of Agricultural Science, p. 107, 1907. 
in mass breeding of as great importance as any that may be hoped to be obtained by looking for a single mutating type evolved through the method of DeVries.

I am unable to affirm whether disease resistance, immunity to disease, is structural or physiological. I believe the latter, for I have been able to develop it in varying degrees of perfection in every strain of potatoes, wheat or flax with which I have worked, and for all of the diseases noted, while the method used has always remained the same. There is also good structural evidence that it is physiological, as shown in the structural changes caused by the entrance of disease-producing organisms within the tissues of resistant hosts. I have worked with pure strains, centgener progeny, from individuals, with the progeny of cross bred plants, with mixed strains and variations in bulk, and with bulk selections of centgener origin, side by side, and resistance has come at approximately the same rate and grade for a variety through any one of these methods. When the conditions of disease production and conditions of infection for each plant have been held so as to be constant factors, the bulk method of selection, as, for example, selection to seed weight, color and form from a disease plot of flax, has given final hereditary resistance as rapidly as individual selections from the progeny of individuals of the same strains. The only cases in which resistance has developed irregularly in such strains of flax or wheat have been found to be due to impurity of type or to irregularities as to the constancy and amount of disease infection, or to irregularities in the conditions promoting the development of disease.

Resistance to the diseases named for the crops named can be developed in any variety or strain by either method of selection noted. In any case, it may be increased by every proper selection year by year, just in proportion to the perfection with which the disease conditions of elimination are maintained.

The method works in potato selection, where the process 
of propagation is by cuttings or buds and hence only a condensed individual life. It works in wheat, which seems quite guarded in its closed or individual fertilization. And it works in flax which, though usually self-fertilized, is, no doubt, much given to intercrossing in the open.

If the proofs rested only upon flax, where there are many possibilities that open crossing might give rise to the various Mendelian types, homozygotes, heterozygotes, etc., the evidence might be thought to be thrown into question, but even there, when crossing is promoted, while there is every evidence of the occurrence of resistant or non-resistant types among the crosses, resistance is seldom found to approach the immune type upon the first possible selection. In other words, if mutants do occur in flax through natural crossing or self-fertilization, so far as our experiments are concerned, these resistant forms are found to act exactly as do those which may be developed from ordinary types of non-resistant flax.

With this crop, one finds little, if any, resistance to wilt and to rust in the best seed strains of southern Russia or in the best seed strains from the new lands of our northwest or in the best fiber strains of the diseasefree districts of northwestern Russia, though it often crops out in the general seed samples from the worst disease infection regions of central Russia. If mutants were without cause and constant, one ought to find them as readily in the seed of one district as in another. One can, however, build resistance upon the least resistant of these strains, if the work is started upon a graded scale of disease infection which is increased year by year. If the seed is placed under too heavy conditions of wilt production, the plants are all killed in embryo or young stages of growth and nothing is gained. If, however, scrubs or runts may be saved from the first season under weak disease infection and a graded infection is followed thereafter, in approximately five years one may bring these strains of seed to such a stage of resistance that ordinary agricultural methods of cropping will maintain 
them upon the most flax-sick areas. But whether these resistant strains have been selected through crossing or by this gradation method from bulk seed or from individuals taken from a known pure strain, I have found that abrupt heightening of disease conditions of too great violence may undo the whole work.

It holds as well for wheat when speaking of rust attacks. Under uniform conditions of rust infection, all wheats arise rapidly to a stage of marked resistance to general uredospore infection whether caused by the type Puccinia graminus or $P$. rugigo-vera, which resistance seems to be characteristic for each variety concerned, but may then fall a ready prey to sudden attacks introduced by properly conducted aecidial infection. All this points to the rôle played by the irritations of environment, which either govern the appearance of mutations or produce other changes which are very worthy of the breeders' and croppers' attention; and allows one to ascribe much more merit to methods of mass selection and breeding in cereals than the DeVriesian doctrine of constancy of elementary species will allow one to assume.

No phase in this argument touches upon the ultimate causes of disease resistance or immunity. But the facts do point quite clearly to the probable influence of chemical agencies, perhaps toxines, arising from the direct existence of fungus attacks upon the hosts. In my mind, there is not the slightest doubt but such attacks originate heritable resistance, in much the same sense as MacDougal's chemical injections upon ovaries are supposed to have originated new types. If later experiments prove MacDougal's observations to be well founded, the results will be of far reaching importance. If these suppositions that fungus attacks upon the host may induce fungusresistant qualities in the progeny from the matured ovaries, are correct, no doubt the unit characters, so called (whether simple and definite in number or whether they may be considered as composed of countless and variable elements of the cromatin structures) may be effected, or 
may be originated, and one ought, under crossing, to observe the effects in terms of dominant and recessive or cloaked features. Such effects I have observed to occur: in flax as against flax wilt and in wheat as against $P$. graminus. Mr. R. H. Biffin, of England, is reported as indicating that this is true in the case of wheat when crossed with eincorn as against yellow rust.

Bateson, in an admirable article upon "Facts Limiting the Theory of Heredity," 12 would also seem to enroll himself as against any adaptative response to changed conditions as able to account for the origin of such facts as I have observed in my cultures of flax and wheat. Thus we read:

"Though the response to change of conditions may have been direct, it must not be hastily concluded that the response is adaptive. The appeal to direct responses so common in evolutionary discussions of thirty years ago, was made to account for the complex adaptations of organism to environment. It is the total want of any evidence supporting that appeal which has driven most of us to disbelieve in the reality of any such claims, and there is nothing in the new evidence, I think, which should shake the attitude of resolute agnostieism which we have thus been led to adopt."

However, to explain such observations as those noted by me in the flax and wheat cultures seems to demand the assumption that additional elements of heritable character arise on account of causes demanding adaptive response. No theory of quantitative subtractions of unit characters already formed would seem to be adequate to account for the observed acquired qualities of resistance. It is probable that only the cytologists are in position to produce direct proof or disproof of the apparently necessary suppositions as to character modifications. Certainly those among them who are experimentally inclined may cut along the line indicated with much hope of uncovering many high lights to breeders. Even here, it may be expecting quite too much that possibly pure physiological qualities should be represented by structural units.

${ }^{12}$ Science, November 15, page 649. 